CAHIERS DE

NARRATOLOGIE

\section{Cahiers de Narratologie}

Analyse et théorie narratives

10.2 | 2001

La voix narrative

\title{
De l'écho aux faux-semblants : analyse de la voix narrative dans Caballeros de fortuna de Luis Landero
}

Dorothée Moulin-Combes

\section{(2) OpenEdition}

Journals

Édition électronique

URL : http://journals.openedition.org/narratologie/10230

DOI : 10.4000/narratologie. 10230

ISSN : 1765-307X

Éditeur

LIRCES

Édition imprimée

Date de publication : 1 janvier 2001

Pagination : 269-283

ISBN : 2914561032

ISSN : 0993-8516

Référence électronique

Dorothée Moulin-Combes, « De l'écho aux faux-semblants : analyse de la voix narrative dans Caballeros de fortuna de Luis Landero ", Cahiers de Narratologie [En ligne], 10.2 | 2001, mis en ligne le 01 janvier 2001, consulté le 11 juin 2020. URL : http://journals.openedition.org/narratologie/10230 ; DOI : https://doi.org/10.4000/narratologie.10230 


\title{
DE L'ÉCHO AUX FAUX-SEMBLANTS : ANALYSE DE LA VOIX NARRATIVE DANS CABALLEROS DE FORTUNA DE LUIS LANDERO
}

\author{
Dorothée MOULIN-COMBES \\ Université Paul Valéry - Montpellier III
}

Un banc situé sur la place principale de Gévora, petite commune d'Estrémadure, voici le lieu à partir duquel le narrateur de Caballeros de fortuna (1994) recueille la matière de son récit. Luis Landero, que la critique espagnole avait plébiscité dès la sortie de son premier roman, Juegos de la edad tardía (1989), nous offre à travers ce second roman les destinées de cinq personnages. Ces personnages pensent tous avoir fait des découvertes susceptibles de changer leur vie. Le professeur Belmiro Ventura retourne au village après avoir pris conscience du peu de temps qui lui restait pour mener à bien ses recherches historiques. Don Julio, un mercier, s'est découvert une vocation politique. L'innocent, Esteban, qui découvre la vie des riches et décide de devenir l'un d'eux. Luciano, enfant miraculeux selon sa mère, découvre l'amour pour Amalia, l'institutrice aimée à la fois du jeune Luciano et de Belmiro Ventura. La fortune (la "fortuna" du titre) va faire tourner sa roue, débouchant sur un dénouement tragique (Esteban, voulant tuer Belmiro Ventura pour une question d'héritage, tue en fait son ami Luciano). Comme dans Juegos de la edad tardía ou dans El mágico aprendiz (1999), troisième et dernier roman en date de Luis Landero, on est plongé dans un monde qui fait la part belle aux aspirations impossibles et aux désirs insatisfaits. Toutefois dans Caballeros de fortuna, à la différence des deux autres romans, l'accent est mis sur la figure du narrateur, sur les mécanismes de la narration, sur les relations entre le narrateur et l'objet narré. Et justement, ces mécanismes et ces relations ne vont pas de soi dans ce roman. Luis Landero se propose de construire un récit qui est problématique du point de vue 
de l'analyse narratologique. D'où l'intérêt d'analyser les structures narratives qui, comme nous le verrons, sont caractérisées par le recours à un faux-semblant narratif. Les marques de l'énonciation jalonnent le texte et ne disparaissent jamais. La voix narrative principale organisatrice du récit qui se fond tout au long du texte dans un "nosotros", s'appuie sur une pluralité de voix autres. Notre propos sera de montrer en quoi cette multiplicité de voix ainsi que la multiplication des points de vue sont au service d'une cause : récupérer et transmettre la mémoire collective de Gévora tout en reconstruisant la réalité, ou pour le moins une réalité. Si le narrateur n'est qu'un écho dans le sens où il emprunte les voix d'autrui, ne laisse-t-il pas par là la porte ouverte à tous les faux-semblants narratifs?

Dans un petit village d'Estrémadure, un narrateur anonyme - une voix qui représente un groupe d'inactifs qui se retrouvent chaque jour sur un des bancs de la place du village $^{1}$ - nous raconte en 1993 une histoire qui s'est déroulée quinze ans auparavant. Pour qui étudie la voix narrative dans un roman, à savoir « l'instance productrice du discours narratif » telle que la définit Genette ${ }^{2}$, il convient de s'intéresser aux relations entre le sujet de l'énonciation et

${ }^{1}$ Dans une entrevue accordée lors de la sortie de Caballeros de fortuna, Luis Landero a déclaré : "Hace tres años hicieron en una revista del MOPU un monográfico sobre plazas en España y me invitaron a escribir sobre ellas. Escribí sobre esos grupos de gente que se sientan en las plazas de los pueblos, jóvenes, viejos, ociosos... que están ahí no se sabe bien por qué y que se enteran de todo, son narradores omniscientes. De ahí me viene la idea de la novela. ", Juan Ángel Juristo, "Luis Landero: "El móvil de la novela radica en la confusión entre amor y poder" ", Leer, n ${ }^{\circ}$ 0, fév-mars 1994, p. 65.

2 Gérard GENETTE, Figures III, Paris, Seuil, 1996, p. 226. Nous privilégierons l'approche narratologique en empruntant à Gérard GENETTE l'appareil critique, devenu classique, utilisé pour mener à bien cette analyse, notamment Figures III et Nouveau discours du récit. Nous ne reviendrons pas sur la distinction auteur/narrateur mais nous renvoyons à ce propos à la première partie de l'ouvrage de Jaap Lintvelt, Essai de typologie narrative. Le "point de vue ». théorie et analyse, Paris, José CORTI, 1981. 
l'énoncé. En effet, réfléchir sur la voix narrative dans un roman revient précisément à se pencher sur les questions de savoir « qui raconte » et « comment il raconte ».

Le narrateur premier de Caballeros de fortuna ${ }^{3}$ apparaît au travers d'une personne du verbe inclusive ("nosotros »). Il est homodiégétique car il est présent comme personnage dans les aventures du village mais il n'influe en aucun cas sur le déroulement de la trame, il n'y joue qu'un rôle de témoin ${ }^{4}$, il n'est pas actant. Il assume le simple rôle de voix narrative et n'apparaît en aucun cas comme un être narré. Il a comme fonction principale, hormis les fonctions narrative et de régie qui sont inhérentes à son statut puisqu'il est celui qui raconte et qui organise le récit ${ }^{5}$, une fonction testimoniale qui « manifeste le degré de certitude ou de distance qu'[il] entretient vis-à-vis de l'histoire qu'il raconte ${ }^{6}$. Luis Landero récupère ainsi un vieux topo littéraire particulièrement efficace dans le genre épique, celui du témoignage oculaire (le fides occuli). Comme en témoignent les nombreuses occurrences des verbes "ver» ou "mirar», le narrateur est un personnage qui observe. Le texte réduit sa fonction à celle de raconter ce dont il a été le témoin oculaire et auditif. Il est souvent réduit à un point de vue " extérieur ", une source, un foyer à partir duquel l'histoire du village est racontée. Plus précisément, c'est un personnage qui observe, écoute puis raconte. Les constructions qui associent au verbe «ver»le verbe " oír " sont récurrentes dans le roman ${ }^{7}$. Une place très importante est accordée à l'oralité, au caractère oral de l'énonciation, de cette fiction qu'est l'énonciation littéraire.

${ }^{3}$ Luis LANDERO, Caballeros de fortuna, Barcelone, Tusquets (col. «Fábula ", $\left.{ }^{\circ} 45\right), 1996$. Toutes les citations renvoient à cette édition et, si besoin, c'est nous qui soulignons.

${ }^{4}$ Gérard GENETTE, Figures III, op. cit., pp. 252-253.

5 Gérard GENETTE, Figures III, op. cit., pp. 261-262.

${ }^{6}$ Yves REUTER, L'analyse du récit, Paris, Dunod, p. 43.

7 Quelques exemples : "y hacia las siete lo vimos aparecer entre los naranjos... Justo en ese momento, oímos acercarse por el camino... » (p. 22), «lo veíamos y oíamos llegar todas las tardes » (p. 42), «lo oíamos avanzar... y lo veíamos ir y venir » (p. 77), "Lo habíamos visto pasar hacia las ocho por la plaza... y habíamos oído... » (p. 96), etc. 
C'est sur ce narrateur voyeur et disert, cette "voz plural » comme la caractérise María José Navarro ${ }^{8}$, que repose tout le roman.

Ce narrateur procède à son auto-représentation comme témoin et ce, dès l'incipit. À l'indéfini de la phrase inaugurale succède très rapidement, dès la fin du premier paragraphe, un « nosotros » qui va jalonner l'ensemble du roman et qui va être très rapidement précisé :

ahora apenas somos media docena, y aquí nos pasamos las jornadas, alineados en un banco corrido de piedra y con los pies mecidos en el aire. El forastero o el curioso no necesita observar siquiera las novedades que se producen a su alrededor; con vigilar los pies es suficiente. Si se mueven, es que algo está ocurriendo, y según el vaivén así el tamaño del suceso ; si enseguida vuelven a pararse, es que se trata de una falsa alarma. La historia de este pueblo, como la de tantos, la han ido escribiendo las generaciones al ritmo de los pies. (pp. 13-14)

Nous citons entièrement ce passage malgré sa longueur car il permet de mettre l'accent sur certains traits caractéristiques de la mise en scène du dispositif énonciateur : à savoir l'importance accordée au banc ainsi qu'aux pieds des témoins ${ }^{9}$. Le lien entre les notations renvoyant aux mouvements des pieds de ces personnages et la narration est souligné par la voix narrative principale à la page 171 par le biais de l'expression «mover locuazmente los pies » ${ }^{10}$ qui personnifie cette partie

8 María José NAVARRO, «Caballeros de fortuna. Metáfora agridulce de la Historia ", Reseña de literatura, arte y espectáculos, $\mathrm{n}^{\circ} 251$, juin 1994, p. 20.

${ }^{9}$ Il y a en tout 18 références aux pieds des oisifs assis sur le banc (9 dans chacune des deux parties du roman). Ces occurrences se trouvent aux pages $13,14,20,22,33,51,134,153,171,182,213,216$, $251,254, \underline{319}$ (les pages soulignées désignent celles où il y a deux occurrences).

10 «Llevaba [Belmiro Ventura] unas dos semanas en el pueblo y nos habíamos acostumbrado a su presencia, o al menos a aquel período ininteligible de tregua, cuando una tarde empezamos a mover locuazmente los pies al verlo tomar al fin el camino de la Levantinita. " (p. 171) 
du corps tout en signalant le caractère synesthésique de la description. Le banc est en quelque sorte une mise en abyme du narrateur. Toutefois, si le banc est le lieu par excellence de la narration soulignant au sens propre la position stratégique du narrateur, il n'en a pas l'exclusivité. Le « casino » remplit à deux reprises cette fonction :

[don Julio] empezó a frecuentar el casino - donde el grupo de observadores nos refugiábamos cuando hacía mal tiempo - (p. 181) ${ }^{11}$

Le pronom personnel "yo », pudiquement compris dans le « nosotros » qui jalonne le roman, est sujet de la narration, du discours. Il est celui qui permet à l'histoire de devenir narration. Il s'individualise et se particularise à une seule reprise dans le roman lorsqu'il s'auto-identifie comme personne :

Yo soy, o era, porque nunca ejercí, maestro de escuela (p. 182)

Mise à part cette indication quant à sa profession, ce narrateur reste anonyme. C'est précisément cet anonymat qui, selon Darío Villanueva, "pretende reforzar su condición comunal $»^{12}$. Il justifie de sa condition de narrateur uniquement en se présentant comme un observateur parmi d'autres. Les autres personnages constituant le «nosotros " ne sont tout au long du roman que des êtres anonymes qui « carecían de otro oficio que el de haber nacido y crecido y estar aquí sentados, observando el mundo y anotándolo al ritmo de los pies » (p. 182). Seules certaines figures se détacheront: l'un des habitués du banc dont on apprend incidemment qu'il est décédé (p. 286), Gil et Gregorio, les deux personnages principaux de Juegos de la edad tardía, qui réapparaissent parmi les observateurs du banc (p. 182) et enfin Belmiro Ventura, l'un des cinq personnages du récit qui intégrera le banc après

${ }^{11}$ L'autre exemple se trouve à la page 191.

12 Darío VillanUeVA, «Caballeros de fortuna », $A B C$ literario, 11/02/94, p. 7. 
la tragédie qui constitue le dénouement du roman (p. 319$320)^{13}$.

Dans Caballeros de fortuna, le type de narrateur témoin choisi par Luis Landero permet une réflexion sur les problèmes de vision. Il faut pouvoir voir pour pouvoir raconter. En effet, les problèmes de voix narrative, interfèrent avec les problèmes de focalisation. L'idée selon laquelle il y aurait une place pour raconter et une place pour agir se fait jour. Celui qui raconte doit voir celui qui agit. La théâtralité du récit est très forte; tout se joue à travers le regard de ce spectateur qu'est le narrateur ${ }^{14}$. Outre le découpage en chapitres qui est à rapprocher des scènes au théâtre, la théâtralité de certaines scènes auxquelles le narrateur a assisté avec les autres habitués du banc est soulignée à l'occasion :

los veíamos pasar otra vez por la plaza al final de la tarde.

Era como si regresaran a escena cuando todos creíamos que habían representado ya completamente sus papeles. (pp. 264-265)

Tout au long du roman le lecteur a l'impression d'assister à une représentation, à un spectacle présenté devant un parterre de privilégiés dont fait partie le narrateur. Toutefois ce qui au niveau de la voix proprement dite (qui parle ? ${ }^{15}$ ) peut sembler fort simple - un narrateur témoin qui raconte une histoire à laquelle il a assisté - acquiert peu à peu une complexité du fait de la multiplication et de la diversité des sources sur lesquelles il s'appuie, c'est-à-dire de la multiplication des voix et des points de vue (qui voit ?). Ces notions

13 Comme l'a fait remarquer Gonzalo Hidalgo BAYAL, on peut rapprocher cette mise en scène de la voix énonciatrice de la "media docena de hombres vestidos con mono, sentados, acuclillados aquí y allá » du Hameau de William Faulkner. Gonzalo Hidalgo BAYAL, "La ficción y el afán (ensayo sobre Luis Landero) ", Cuadernos hispanoamericanos, $\mathrm{n}^{\circ} 535,1995$, p. 120.

14 Le terme de "espectadores" se trouve à la page 251.

15 « relève de la voix, non seulement le statut du narrateur, c'est-àdire ce qui constitue la réponse à la question «qui parle ? », mais aussi les problèmes du temps de la narration et ceux des différents niveaux narratifs, du récit dans le récit. ", Mieke BAL, Narratologie, Paris, Klincksieck, 1977, p. 21. 
convoquent d'autres problèmes, notamment les relations que le narrateur entretient avec ce qu'il raconte. Qui plus est cette structure a le mérite d'induire une réflexion sur le réel, sur la vérité étant donné que la réalité est construite à partir de témoignages recueillis par le narrateur et qui sont autant de discours, donc de reconstructions, de cette réalité. Un des intérêts de ce dispositif narratif est de mettre l'accent sur la médiation de ce narrateur.

Le récit du narrateur premier constitue en fait un texte porteur comprenant d'autres récits, c'est-à-dire qu'il englobe différents récits sur lesquels s'appuie le narrateur lorsqu'il délègue parfois sa parole. La production de ce narrateur est de fait «un discours habité par des énonciateurs multiples » pour reprendre une formulation de $\mathrm{C}$. Boix ${ }^{16}$. Les rapports entre la voix narrative principale et les autres voix du récit sont donc essentiels. Le narrateur reconstruit la réalité à partir de sources de nature hétérogène : récits oraux, documents écrits (lettres d'Esteban, journal intime d'Amalia ${ }^{17}$ ), observations, rumeurs, suppositions. Les sources sont donc multiples. Les points de vue sont variés. S'instaure ainsi un jeu de premières personnes imbriquées. Certains des personnages principaux peuvent en être à l'origine comme don Julio, le « cronista local » (p. 13), Belmiro Ventura, Amalia ou des personnages secondaires qui n'ont pour fonction dans le récit que celle de témoins permettant de combler certains vides et d'accroître par là même la cohérence et la logique interne du récit comme par exemple le curé Juan Mirón (confesseur de Luciano), María la Bonita ou encore le banquier. Même s'il ne

${ }^{16}$ Christian BOIX, «La voix des personnages. Pepe Carvalho dans Los mares del Sur, de Vázquez Montalbán ", Le personnage romanesque, Cahiers de narratologie $\mathrm{n}^{\circ} 6$, Nice, Université des lettres, 1995, p. 155.

17 « en la mudanza que mandó hacer [Amalia] se traspapelaron sus diarios, y algunos sucumbimos a la tentación de leerlos -como también llegamos a leer las cartas que Esteban y Luciano le escribieron a Sofía Sánchez, y que un día aparecieron devueltas y sin remite en la estafeta de correos, y todas con una nota manuscrita donde constaba que el destinatario ya no vivía allí. », p. 320. 
disparaît jamais véritablement, le narrateur premier extradiégétique peut disparaître de façon momentanée, cédant la place à un personnage qui, en se mettant lui-même à raconter une histoire, va devenir narrateur intradiégétique, augmentant ainsi l'« illusion référentielle » (Riffaterre). Certains critiques, entre autres Darío Villanueva, ont souligné l'artificialité d'une telle mise en scène visant à justifier les sources du narrateur :

Landero se ha dejado seducir por los « escrúpulos del punto de vista » que exigen al narrador justificar en cada caso el origen de lo que narra. [...] aquel narrador que, como el de Balzac, se asemeja a un jefe de policía que tuviese acceso, incluso, a los archivos de la Providencia. ${ }^{18}$

Il convient cependant de remarquer que la "vérité » (romanesque), contrairement à ce à quoi on pourrait s'attendre, ne jaillit pas de la confrontation entre les différentes sources. Elle s'apparente à une reconstruction, le terme apparaît précisément dans le roman :

Y aquí, donde tarde o temprano todo acaba sabiéndose, hemos reconstruido más de una vez aquella historia que ocurrió hace 15 años, y de la que ya apenas nada queda por contar. (p. 320)

Le narrateur et ses acolytes reconstruisent la réalité à partir des différents éléments qui leur sont fournis :

Nadie podía imaginar por entonces que aquellos episodios eran piezas sueltas que acabarían por combinarse para formar una sola historia, ni que cada personaje, con sus actos insignificantes de cada día trabajaba ya para un riguroso desenlace común. (p. 213)

Ils mettent en ordre les informations obtenues de différentes sources afin de reconstruire la chronologie des événements. S'il y a polyphonie énonciative, le pluriperspectivisme auquel pourrait se prêter parfaitement ce type de construction narrative et que nous trouvons par exemple dans Muertes de perro de Francisco Ayala est, dans Caballeros de fortuna, très

18 Darío VILlanUEVA, « Caballeros de fortuna », art. cit., 11/02/94, p. 7. 
limité19. Le texte nous en offre toutefois un exemple à travers les versions d'Amalia et de Luciano sur leur relation amoureuse :

Así se lo contó Luciano en el confesionario al padre Mirón, y así lo cuenta Amalia en sus diarios. (p. 210)

Mais, comme nous pouvons en juger, ce perspectivisme est ici tout à fait relatif du fait de la concordance des sources - concordance soulignée par le parallélisme syntaxique. La confrontation n'est pas porteuse d'un questionnement sur le réel, ni matérialisation narrative de l'ambiguïté de la réalité.

À propos de ces différentes sources il convient de réfléchir à la façon dont elles sont retranscrites. En effet, plusieurs possibilités allant de la scène au résumé s'offrent aux narrateurs premier ou second. Leur combinaison est bien sûr grandement exploitée. Qui plus est, tout un travail sur l'hébergement de la parole d'autrui se fait jour. Le recours à la citation et à la reproduction des propos de certains personnages présente l'intérêt d'illustrer différentes modalités du discours rapporté : discours narrativisé au style indirect, discours transposé au style indirect libre, discours rapporté au style direct. Si nous prenons l'exemple des informations données par María la Bonita à propos d'un fragment de la vie d'Esteban, nous voyons que ce personnage féminin devient producteur d'un métarécit ${ }^{20}$ et que deux modalités du discours sont privilégiées : le discours narrativisé intégré au récit de ce narrateur et le discours rapporté. Le " contaba una mujer, María la Bonita de nombre, que... » révèle le fonction-

19 Une des grandes différences entre ces deux romans du point de vue qui nous occupe ici est que dans Muertes de perro le lecteur doit procéder activement à la reconstruction de la réalité à partir des différentes versions de celle-ci alors que dans Caballeros de fortuna le lecteur accepte que ce travail ait déjà été effectué en amont par le narrateur premier.

${ }^{20}$ Le passage se trouve aux pages 121 et 122 . Ce discours au style direct offre l'avantage de proposer deux niveaux: les paroles de María la Bonita et celles d'Esteban qu'elle-même cite (la typographie signale également que nous sommes à un autre niveau de narration). Ce passage fait la part belle à la scène, toutefois le sommaire et l'ellipse sont également utilisés. 
nement du texte. En effet, l'indéfini total de l'imparfait permet de décontextualiser le récit, de créer un relais narratif. Toutefois les conditions de possibilité de la narration de ce personnage font défaut. Nous sommes en présence d'un récit décatégorisé21. Par cette option, le narrateur ne brouille-t-il pas la logique du récit intradiégétique ? C'est précisément dans la façon dont il agence les différents récits que réside l'originalité de ce roman. Il est parfois difficile d'affirmer dans Caballeros de fortuna si nous sommes en présence d'un premier ou d'un deuxième niveau de narration. En effet, si le narrateur premier peut se désengager, il se contente la plupart du temps de faire parler un personnage tout en restant le producteur du récit.

Globalement dans Caballeros de fortuna le narrateur se limite à la responsabilité du récit. Il prétend rapporter objectivement les faits tels qu'il en a pris connaissance (« un grupo de observadores imparciales » [p. 13]). Cependant, certaines notations traduisent la subjectivité du narrateur. Il s'agit pour la plupart de réflexions sur l'écriture d'Amalia dans ses journaux intimes :

Pero ya no era la misma, y así lo confiesa en sus diarios, donde hay desde entonces un temblor de incertidumbre (p. 212-213)

Ou encore :

Y todo eso lo iba escribiendo de un tirón, a juzgar por la descompostura de la letra (p. 304)

Un autre jugement du narrateur faisant montre de sa fonction évaluative est manifesté à propos de don Julio par la notation entre parenthèses :

Carraspeaba mucho (hasta escribiendo se le notaba), y cuando al fin enhebraba una frase, enseguida aislaba una palabra (p. 182)

Nous remarquons que dans ces exemples plus que l'instance narratrice c'est l'instance lectrice qui s'exprime.

21 Nous entendons par là un récit extérieur à des catégories de temps, d'espace et d'action. 
Un des intérêts de cette structure est de mettre en évidence la construction même du discours romanesque ce qui génère une grande complicité avec tout lecteur ayant accepté le pacte de lecture proposé par le narrateur. En effet, le discours romanesque se constitue à partir d'autres discours. Aussi peut-on se demander si l'on ne peut voir dans ce deuxième roman de Luis Landero un dévoilement du travail sur les matériaux, caractéristique de l'art romanesque. Ce que l'on nous montre, ce serait une création en train de se faire.

Dès la deuxième page du roman, le narrateur premier de Caballeros de fortuna auto-qualifie sa narration de chronique :

De tanto golpear, el banco tiene abajo una franja erosionada y sucia, y allí a su modo está esculpida, como en un bajorrelieve, la crónica ilegible y exacta de nuestro pasado cotidiano. (p. 14)

Et c'est bien d'un narrateur chroniqueur dont il s'agit puisque sa narration consiste en un témoignage oculaire et en la référence à une multiplicité de sources. À trois reprises le terme de " chroniqueur » est employé par le narrateur pour qualifier les personnages du banc dont il fait partie. Nous n'en donnerons qu'un exemple qui souligne en outre une des caractéristiques de la chronique, à savoir son dessein premier qui est d'enregistrer les différents événements pour pouvoir ensuite les transmettre en faisant acte de mémoire :

y luego retomábamos nuestro compás unánime de cronistas de la actualidad (p. 255) 22

Comme le fait remarquer un critique espagnol, Caballeros de fortuna « es el producto de una crónica colectiva ${ }^{23}$. Ainsi le narrateur s'apparente-t-il à un mémorialiste qui tend à récu-

22 Les deux autres exemples se trouvent aux pages 15 («Pero, desde aquel día, han pasado ya más de quince años, y algunos de los cronistas han muerto y otros deben de andar sabe Dios por qué tierras. ») et 109 («los cronistas del banco verían ir o venir »).

23 Juan Antonio MASOLIVER RÓDENAS, « Luis Landero. La lógica del ensueño ", El Urogallo, n 95, avril 1994, p. 60. 
pérer et transmettre cet "espíritu de nuestra época » dont parle le texte (p. 287). Le moment de la narration comme en témoignent de nombreuses indications se situe en 1993 :

Hoy vuelve a ser junio, día 5 de 1993, y mañana habrá elecciones generales. (p. 321)

Le récit analeptique, pour sa part, fait référence essentiellement aux années 1976-197824:

Y era allí hacia donde mirábamos aquella tarde de octubre de 1977, esperando que apareciese algún automóvil para poder comprobar, aunque sólo fuese fugazmente, hasta qué punto había envejecido Belmiro Ventura después de tanto tiempo de ausencia.

Pero, desde aquel día, han pasado ya más de quince años (pp. 14-15)

La portée de l'analepse est ici mise en évidence explicitement par la précision « han pasado ya más de quince años » ou de façon plus approximative par l'usage de déictiques signifiant l'éloignement. Tout au long du roman nombreux sont les exemples où à un "ahora " correspondant au temps de la narration s'oppose un «aquel tiempo » ou un «aquel día » référant au temps de l'histoire ${ }^{25}$. Le jeu sur les déictiques matérialise le décalage temporel. Le narrateur, depuis le présent de son instant d'élocution, fait œuvre de mémoire à travers cette narration ultérieure qui lui octroie une supériorité par rapport aux faits qu'il relate :

era imposible saber en aquel tiempo que no se trataba de Dios, ni de ellos, sino del mero destino (p. 87)

Force est de remarquer que Caballeros de fortuna a en commun avec la chronique, prise dans son acception de

24 Toutefois quelques analepses de portée plus importante renvoient aux années antérieures comme par exemple l'épisode de la folie de Manuel, le père d'Esteban, après la mort de son autre enfant (second chapitre de la première partie).

25 «Ahora, en estas tardes de entrega incondicional al tiempo, sin otra expectativa que la llegada de un forastero o del anochecer, Belmiro Ventura accede a recordar que aquel día, y por primera vez en su larga carrera docente, pretextó una indisposición para no entrar en el aula »(pp.115-116). 
recueil de faits historiques, le respect de la chronologie des faits, qui sont rapportés globalement dans l'ordre de leur succession. Le rapport entre chronique et temporalité est fort intéressant. En effet, la chronique fait passer la personne du temps réel et objectif au temps narratif. Ce roman est bien une chronique dans le sens où il s'agit d'une compilation des informations qui circulent - ou tout du moins ont circulé dans le village. Et le travail du narrateur est bien un travail de compilateur. Qualifier un récit de chronique n'est en rien innocent. Cela contribue à créer l'illusion de réalité, cet « effet de réel » dont parle Barthes, car dès lors qu'il s'agit d'une chronique, l'œuvre réfère à un hors-texte. De plus, un des avantages de la disparition momentanée du narrateur premier permet d'accroître l' «illusion référentielle » tout comme la mention des documents écrits connote l'authenticité des dires.

Le quotidien devient discours. Il est narrativisé à travers cette récupération de la mémoire collective du village. Pour reprendre un thème cher à Juan Marsé, il s'érige en sujet d' " aventis » 26 . Le banc est dès lors métaphoriquement le lieu où s'écrit l'histoire du village au sens propre (cf. le bas-relief) comme au sens figuré. Il convient de remarquer que la chronique se doit d'être objective tant dans l'observation de la réalité que dans sa retranscription. Et c'est en ce sens que l'on doit reparler du "nosotros » que l'on a analysé précédemment. En effet l'utilisation du «nosotros » induit une désubjectivisation du discours. Pourquoi le narrateur choisit-il une première personne du pluriel et non du singulier si ce n'est pour que le moi s'efface ? Ce pronom personnel, hormis le fait qu'il permet d'éviter toute tentation de subjectivité, tend à prouver qu' on joue sur les voix narratives. Toutefois l'objectivité cède parfois le pas à la subjectivité. Un glissement se produit souvent entre ce qui a été et ce qui a pu être. Pour certaines pièces manquantes à la reconstruction de la réalité, le narrateur fait parfois appel à l'autorité fort questionnable de cet actant particulier qu'est la rumeur et à des suppositions souvent matérialisées par des constructions du

26 Ce rapprochement n'a rien d'anodin puisque l'institutrice, Amalia, lit Últimas tardes con Teresa. 
type « deber de ». Mais n'est-ce pas là précisément une des caractéristiques de la récupération du passé par la mémoire collective que de combler les lacunes du souvenir par des extrapolations.

L'histoire que nous propose le narrateur de Caballeros de fortuna est donc le fruit d'une "reconstitution fragmentée obtenue par réajustements mémoriels ${ }^{27}$. L'instance narratrice met en perspective un passé récent, celui des années suivant la mort de Franco. Les aspirations des protagonistes se font l'écho des espoirs de la société espagnole. Le narrateur est le dépositaire de la mémoire collective de Gévora. Il est un des maillons de la transmission de ce savoir populaire ; il n'est en aucun cas le garant de tous les savoirs du village. En effet, le recours aux discours des divers personnages contrebalance la voix narrative principale.

Nous conclurons en rappelant que tout au long de Caballeros de fortuna la narration est mise en évidence du fait même de l'importance accordée à la figure de ce narrateur chroniqueur. Le dispositif narratif qui s'appuie sur cette « voz del narrador, de resonancias casi ancestrales $»^{28}$, ayant pour mission de transmettre l'information, l'apparente par certains aspects à la tradition folklorique. Le narrateur et les personnages sont des conteurs. Les nombreux «cuentan que » ou des formulations telles que «tal como lo oí te lo cuento a ti » (p. 266) ne se contentent pas de souligner la dimension orale du récit mais désignent également l'activité narratrice. En intégrant le banc qui, selon lui, "no era un mal sitio para un historiador » (p. 319), Belmiro Ventura entre en narration : il donne vie oralement à l'œuvre qu'il n'a pas écrite. À travers

27 Nous empruntons l'expression à Georges Tyras, «Roman espagnol contemporain et déni de l'histoire: de Muertes de perro à Beatus ille ", Sociocriticism, vol. X, 1-2, n 19-20, 1994.

${ }^{28}$ Santos Sanz Villanueva, «Vuelta de tuerca hacia atrás », Diario 16 (sup. «Culturas »), 12/02/94, p. 14. 
l'anamnèse, le chœur antique ${ }^{29}$ des observateurs dont fait partie le narrateur donne à voir le passé de Gévora tout en mettant en évidence la construction même du discours romanesque. Ces personnages assis sur le banc ne peuvent-ils être perçus comme une mise en abyme de l'activité de lecture ? Et ce, d'autant plus que le narrateur signale à maintes reprises la complicité instaurée avec le lecteur notamment à travers l'emploi du pronom "nosotros " incluant le lecteur et ne renvoyant plus uniquement au groupe des observateurs (p. 227) ou encore avec des phrases qui mettent en relief le parallélisme entre la position du narrateur, en attente d'informations, et celle du lecteur :

« ¿Qué habrá escrito ahí con tanto ardor nuestro buen cronista ?, se preguntarán ustedes », decía [don Julio] al final [...]. « Pues bien, complaceré su curiosidad. » Y nos complacía. (p. 185)

De plus, le prétexte de la chronique transforme l'observateur en narrateur alors que lui, et les autres personnages du banc, sont essentiellement des narrataires. La citation précédente met l'accent sur la notion de plaisir ( nos complacía »). Or, il ne faut pas oublier à quel point le rapport entre désir et plaisir est fondamental dans la relation de communication littéraire : le narrataire se trouve dans une situation de désir que le narrateur est censé satisfaire ou pas. Nous parlions en introduction des "aspirations impossibles » et des " désirs insatisfaits " comme thématique du roman et cette citation tend à prouver que, dans cet univers de frustrations, seul le plaisir narrataire peut être satisfait. Voilà la leçon littéraire que nous donne Caballeros de fortuna.

29 L'expression a été employée notamment par la presse française lors des recensions du roman lors de sa parution en traduction ( $L e$ Monde du 14/11/97 et Le Nouvel Observateur du 23-29/10/97). 